\title{
Constructing meanings for formal use of mobile communication applications in educational contexts
}

\author{
Ewerton M. de Menezes \\ Instituto de Computação \\ UNICAMP \\ Campinas - SP, Brasil \\ ewerton89@gmail.com
}

\author{
Heiko Hornong \\ Instituto de Computação \\ UNICAMP \\ Campinas - SP, Brasil \\ heiko@ic.unicamp.br
}

\author{
M. Cecília C. Baranauskas \\ Instituto de Computação \\ UNICAMP \\ Campinas - SP, Brasil \\ cecilia@ic.unicamp.br
}

\begin{abstract}
Mobile communication applications such as WhatsApp, Facebook, and Twitter, are already present in educational contexts, although their use is generally restricted to the original purpose of informal communication. In this paper, we investigate their potential to promote interaction in formal communication processes, exploring the meaning evolution of WhatsApp use in an undergraduate class setting. Based on an Organizational Semiotic analysis, we proposed a system to enable students to perform preparatory pre-class activities with WhatsApp exchanging commands, text and multimedia messages. Our analysis of the system use suggests that it is feasible and effective to appropriate mobile communication applications which students are already familiar with and use them in formal educational contexts.
\end{abstract}

Keywords - Mobile learning; WhatsApp; Communication and collaboration; Constructing meanings

\section{INTRODUCTION}

Mobile devices such as smartphones and tablets are already present in educational contexts. The use of these devices has the potential to increase participation and engagement inside and outside the classroom, besides increasing the interaction between students and teachers, enabling new forms of teaching and learning [1]. Solutions used in this context include applications specially developed for mobile devices, mobile versions of applications originally developed for desktop or the Web, and general-purpose mobile applications, for example, communication tools like WhatsApp, Twitter, and Facebook. Each category has its own design and evaluation challenges.

In this work, we focus on general communication applications, such as, WhatsApp, Twitter, Facebook, Slack and Telegram, that students already love and spend a lot of time on them, thus not requiring learning how to use new tools. By using this approach educational administrators and professors can engage with students in a medium that is ubiquitous, familiar and conformable for everyone.

The online content created in the formal context of teaching and learning activities (such as textual discussions or links, photos, and video sharing) can be used in the classroom in different ways depending on the strategies applied by students and teachers. The amount of data generated by a class during such activities is often too small to employ Artificial Intelligence techniques and methods, such as natural language processing techniques and machine learning, for generating good quality synthesis, ratings, etc. Usually a corpus much bigger than, for example, 500 words produced by 50 students would be necessary. However, this volume of unstructured content is large enough to burden or inhibit the usage of manual strategies (e.g. reading or assessing all content, synthesis or classification); or semi-automatic strategies (e.g. using word clouds, copy, and paste content).

One possible solution is to adopt generic communication applications combined with some formal elements of communication. The formality brings well-defined structure, rules, protocols and procedures, which in turn facilitate the usage of content generated by students in class. As an illustration, different from an informal statement like "discuss and comment", a more formal statement like "list three positive points, three negative points and suggest improvements" leads to content much structured therefore easier and faster to handle.

With the recent proliferation of chatbots, these messaging platforms have the potential to be used for lots of tasks beyond just communicating with friends. For instance, now it's possible to receive whether forecasts, book a doctor's appointment, ask for an Uber, or shop online just by exchanging messages with chatbots. Some chatbots have sophisticated artificial intelligence and machine learning algorithms behind them, others have databases of information associated with automated responses. However, the adoption of a tool that makes sense and is primarily used in informal contexts in a new formal context is not straightforward.

One need to address at least two questions when chatbots are introduced in the educational context: How do stakeholders "make sense", i.e. adapt the pre-established meanings for these communication applications from the informal to the new formal context? What should the system be like to support these applications in a formal educational context? In this work, we explore these two questions in a real context where WhatsApp is used in an undergraduate course of Computer Science.

We designed the first version of a system that receives messages from WhatsApp and presents them in a form that allows a smooth integration with the course's online platform. 
We conducted a case study using the system in the first semester of 2015 during two class activities of an HCI (Human-Computer Interaction) lecture at the University of Campinas (UNICAMP), in São Paulo State, Brazil. We defined two pre-class activities, in which students interact with the system through textual and multimedia messages sent from WhatsApp. Our analysis of usage suggests that the proposal is viable and the interested parties construct meaning for the formal use of the WhatsApp.

This article is organized as follows: the next section presents related work, in the sequence, one section clarifies the problem, and the other section presents the case study and its results; finally, a section discusses the results and the conclusion is presented.

\section{RELATED WORK}

The pedagogical context we targeted to investigate the informal meanings of WhatsApp was an undergraduate lecture from Computer Science course which employed active learning techniques in the class dynamics. Active learning approaches stimulate students to do more activities than just passively receiving content and information from the lecturer [2]. More specifically, students do preparatory exercises, also called warm-ups, carried out online before class. Based on the content generated by students in these warm-up activities, the teacher can fine-tune the class according to students' needs, in a dynamic inspired in Just-in-Time Teaching (JiTT) [3].

The market already presents well-established solutions for usage of mobile devices combined with active learning. Such solutions include Learning Catalytics [4], a Classroom Response System (CRS), which implements active learning inside the classroom through conceptual tests about the class material, and Blackboard that, besides being a CRS, functions as a Learning Management System (LMS) [5], used to publish files, asynchronous communication and access to online content. Even though these solutions treat satisfactorily the technical and formal levels of this educational context, they do not integrate effectively with the daily life of students and teachers, i.e., the informal level of the educational context, in the manner that mobile communication applications and mobile social networks currently do.

The growing popularity of smartphones in formal and informal educational contexts make possible new means of communication and collaboration between students and teachers. Mobile applications such as Twitter, Facebook, Google Hangout e WhatsApp deliver different functionalities and levels of accessibility that influence their adoption for pedagogical purposes [6].

Institutions that integrated mobile device and social networks, such as, Twitter and Facebook in pedagogical activities in higher education observed the possibility of more personalized and authentic learning for students [7]. It was also noticed that mobile devices could bridge the gap between formal and informal learning opportunities, generating a positive impact on engagement and knowledge sharing [8]. However, there is still a certain resistance in their usage, because these devices are often perceived as a distraction to regular pedagogical activities, mainly due to the informal nature associated with their usage.

Prestidge [9] informally incorporated Twitter in class activities, without any mandatory task associated. The teacher encouraged the students to share comments on Twitter during class about content seen in class, and outside the classroom, to post questions for discussions. The teacher also reviewed the messages posted on Twitter raising questions for class. In that study, Twitter was used with its original communication purpose, and the content created by students was not easily accessible for teacher's use in class.

Kassens-Noor [8] also explored Twitter as an informal tool for active learning applying it to perform assignments outside the classroom. The study compared the usage of Twitter against user diary and face-to-face discussions. Although considered a powerful collaboration tool for students to foster knowledge creation in groups, the 140-character limit offered a barrier for critical thinking and self-reflection, both essential active learning attributes.

Despite being released in 2010, WhatsApp is still a relatively new tool for education. It is possible to find similar strengths of previous technical solutions, however, WhatsApp presents some new features that encourage teachers and students adoption [6], [10], [11]:

- Different from other mobile applications, e.g. Twitter and Facebook, WhatsApp was conceived for the mobile platform;

- It has accessibility features that provide a better interaction for people with disabilities, such as support to increase the font size;

- The Internet consumption is greatly reduced, and some service providers offer specific data packages.

Bouhnik [10] analyzed the meanings and usage of WhatsApp groups in high school courses. The study identified the following main purposes: (1) communication with the students; (2) incentive of a social atmosphere; (3) dialog creation and encouragement of the exchange of experience among students; (4) as a learning platform. In spite of advantages from the technical, educational and institutional perspectives, the large flow of messages within these groups make the maintenance a time-consuming task for teachers.

Rambe [12] further explored WhatsApp groups placing more emphasis on the perception of students, whom over the semester of a university course from Information Technology were divided into WhatsApp groups that included the teacher. Students were encouraged to answer questions posted by the teacher and, in addition, to generate and discuss new questions about class topics. Compared with discussions promoted in the classroom, the WhatsApp groups fomented a socioconstructivist environment in which students help one another in an informal manner. Furthermore, the usual anonymity of the online interaction allowed that shy and less confident students also engage productively in the discussions; similar results can be found in [7]. 
In higher education classes that adopt the LMS Blackboard as an institutional tool and WhatsApp as an informal communication tool, Bere [11] reported that students preferred interact and collaborate using WhatsApp.

The applications of chatbots in mobile learning can be transformative and help students in many domains, such as, coaching, reference guide about a specific subject, performance support, practice and assignment, simulations, or moderator in social learning setting. Fadhil [13] combined conversational UI and gamification elements in a chatbot specialized in teaching children about healthy lifestyle developed for Telegram. Moreno [14] created a chatbot that answers questions about Linguistics also using NLP in the WhatsApp. Both works the authors used the chatbot to convey a specific knowledge and were not customized by the teacher.

Most of the educators that employ communication and social networks applications for mobile devices in educational settings are somewhat limited to explore only the informal use of these tools without changing their original purpose. Moreover, studies in which mobile tools have undergone shifts in the meaning from informal to formal implicates a heavy workload for teachers. This overwork could be lessened if a system could be designed in the technical level of the problem to facilitate the integration of mobile devices in the class dynamics.

\section{PRoBlem ClarificATION}

In order to better understand the impact of mobile technology and applications, mostly used for entertaining in informal contexts, when they are applied in formal activities, we conducted an analysis using three artifacts from the Organisational Semiotics (OS): Stakeholders Diagram [15], the Evaluation Frame [16] and the Semiotic Ladder [16]. The Stakeholders Diagram makes explicit the stakeholders directly or indirectly involved in the problem and consequences of its solution. The Evaluation Frame extends the Stakeholders Diagram supporting the stakeholders to anticipate solutions to problems in the early stages of the design process. The Semiotic Ladder organizes the system requirements into six layers of information; whereas the top three are related with human information functions, the bottom three are related to the technological platform.

The Stakeholders Diagram in Fig 1 showed that not just students and teachers were interested parties in the solution, but also teaching assistants, other mobile communication applications, smartphones and tablets manufacturers, LMS/LCMS companies, along with university's administrative departments, to mention a few.

The Evaluation Frame in Fig 2 illustrated problems and questions different stakeholders grouped in the Contribution, Source, Market, and Community layers from the Stakeholder Diagram could face, along with possible ideas and solutions. The problems and questions raised encompassed the technical, formal, informal and social layers of the mobile active learning, such as how to handle poor Internet connection and service unavailability, how to deal with students without smartphones or overcrowded classes, and privacy concerns about students using their own device for academic activities.

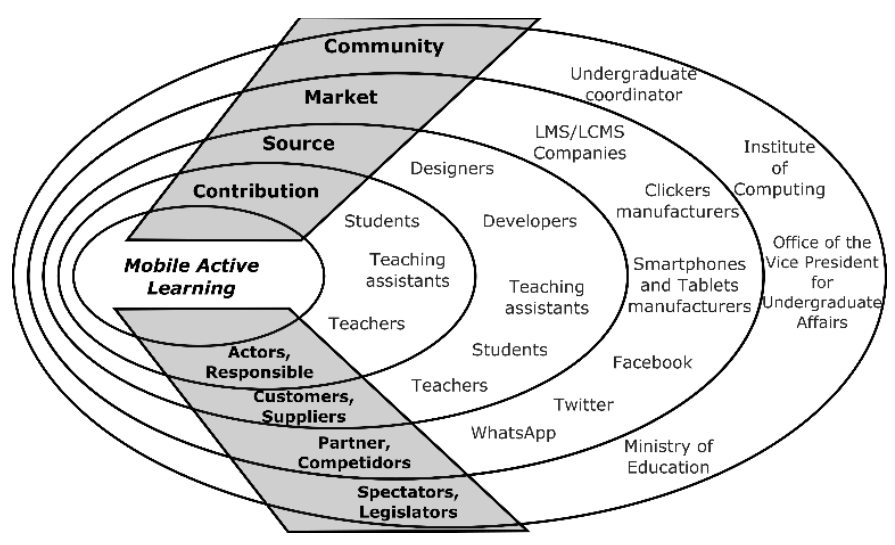

Fig 1. Stakeholder Diagram instantiated

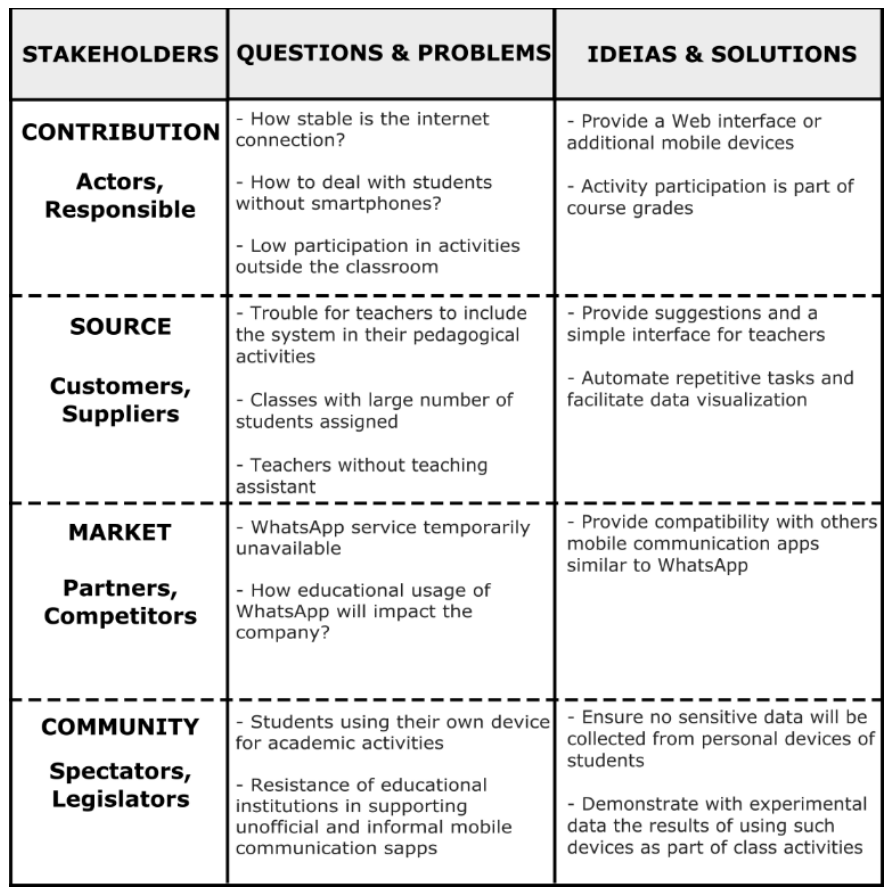

Fig 2. Evaluation Frame instantiated

The system requirements were organized throughout the Semiotic Ladder as shown Fig 3. The Social World and Pragmatic layers bundled the high-level goals and functionalities of the system. The Semantic and Syntactic layers mapped the elements that influence an interaction model based on textual commands, feedback messages and the multimedia information that can be used with mobile communication applications. Lastly, the Empiric and Physical World layers assembled the actual devices, sensors and infrastructure necessary to materialize such a system. 


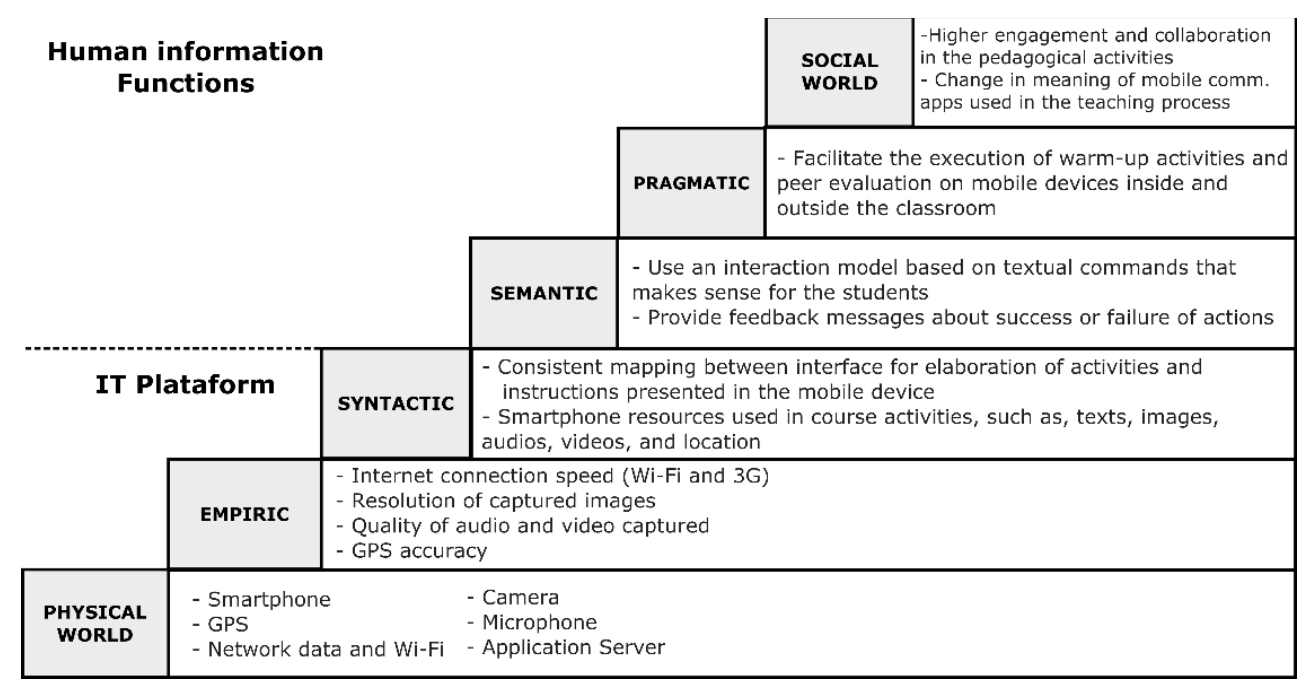

Fig 3. Semiotic Ladder with system requirements instantiated

\section{CASE STUdy}

An exploratory study was conducted comparing the students' participation and contributions during warm-up exercises before lectures, using the system proposed, and the Web interface of the lecture's wiki. We were interested in observing the students' behavior and the impact of the usage of an informal mobile communication application in the formal activities of the lecture. Would the type of interface used to perform the warm-up exercises affect the participation rate? Would the type of interface affect the quality and size of texts written by students? Would the type of activity conducted in the warm-up exercises influence the usage of WhatsApp with the system proposed?

\section{A. Scenario}

The study was conducted throughout a semester in an HCI lecture at UNICAMP, attended by 37 undergraduate students. The warm-ups activities performed by students during the semester involved various types of collaborative activities, such as discussion topics, collecting information as well as questions creation and improvement. The students' participation in the activities were considered in the final grade of the course.

\section{B. Technological Environment}

The technical environment is composed of the integration of a WhatsApp API, which allows sending and receiving text and multimedia messages over WhatsApp's network, with a software developed in PHP named "mAssignments". This software contains the instructions and information required to perform the warm-ups, registers the messages exchanged by students and provides the results in Web pages that can be accessed directly, or embedded in the Wikispaces Classroom, the platform for online collaboration and communication used in the course.
The architecture in Fig 4 is composed of three main elements. The WhatsApp network in which the students can send and receive messages from their mobile device through Wifi or $3 \mathrm{G}$ connections. The online platform used by teachers and students to access the course material and the content produced in the warm-ups. Connecting these two elements, the mAssignment system combines the instructions and rules defined by the teacher for each warm-up with formal elements of communication, such as commands, markers, feedback messages, etc.

The WhatsApp API allows to register a telephone number and respond to multiple events which happen in the WhatsApp network related to that number. Using the API, it is possible to send and receive text messages, multimedia messages (such as images, audios, localizations, and videos), creation and management of groups, besides tracking contact statuses. To interact with mAssignment, the student needs to add the system's number to the smartphone contact list, as shown in Fig 5. The interaction is based on textual commands and markers inspired by social networks, like Twitter, Facebook, and Instagram. To make more apparent that the students were interacting with a system and not with a real person, the system's profile picture is a QR Code instead of the default photo, and the course name and code were placed in the contact information fields.

Warm-ups currently supported by mAssignment can be classified into two categories. The first one refers to warm-ups of information collection, that encompasses activities in which students are stimulated to go into the field with their smartphones and capture images, audios and videos, with or without geolocation information. The second one refers to warm-ups that involve peer evaluation, i.e., students evaluating each other's work via WhatsApp. 


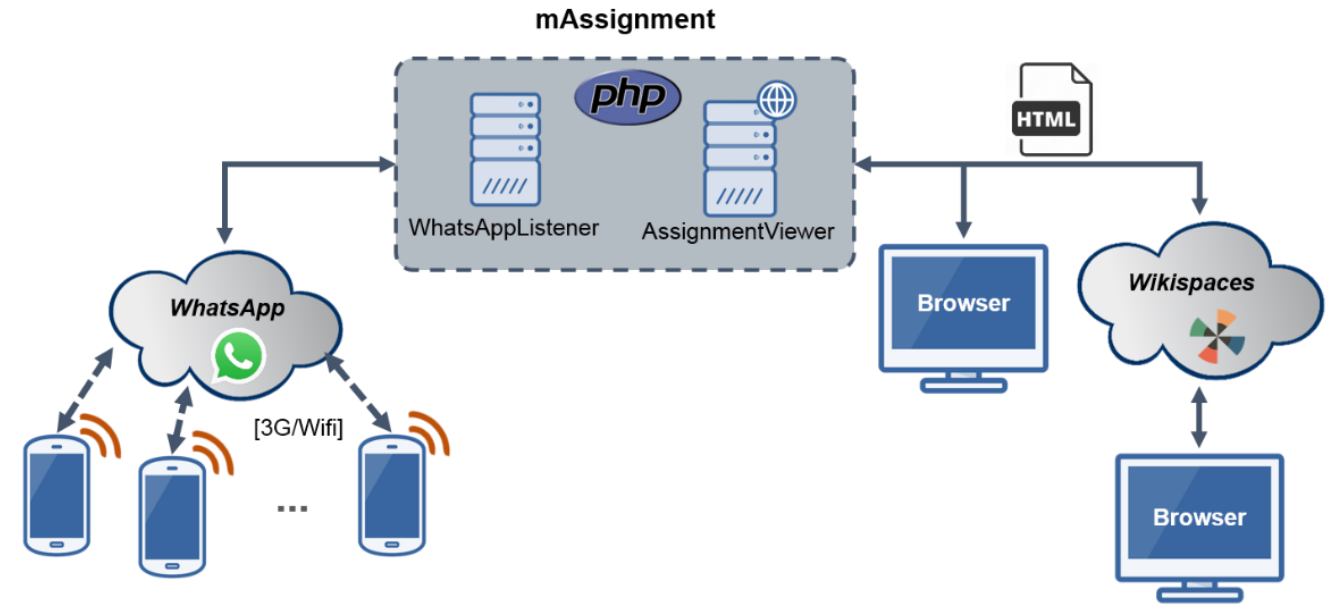

Fig 4. Architecture overview of the mAssignment system

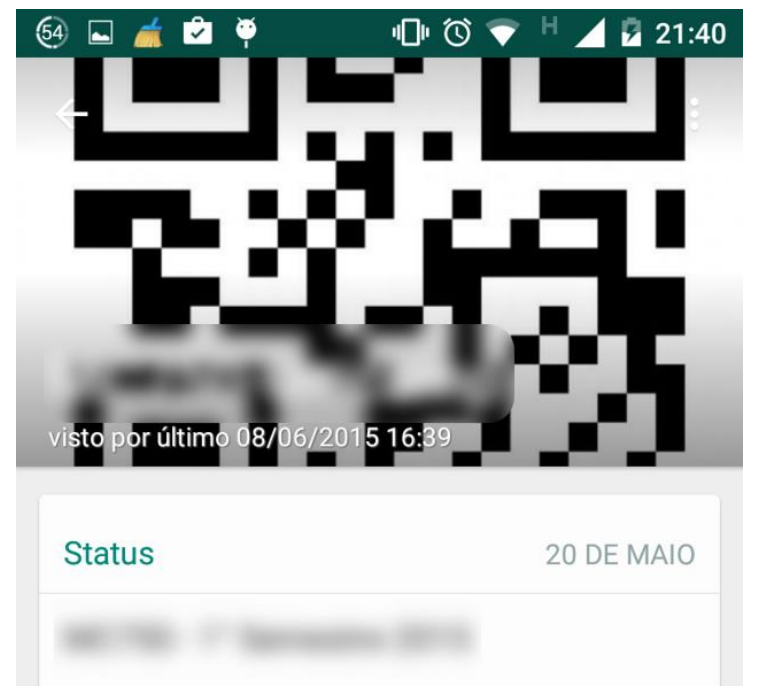

Fig 5. System's contact added to the contact list

The basic dynamic of a warm-up is composed of five steps; foremost the students select which warm-up to start by using the command: “@<Warm-up_code >”(e.g., @AQ12,@AQ13, etc). The second step, students need to identify themselves by sending their course login with the command “@<Course_login>”, so no previous registration involving the phone number is necessary. In the third step, the students select, from a pre-registered list, the group to evaluate with the command "@<group_name >”; note this step is required only in peer evaluations. In the fourth step, students perform the warmup according to the instructions defined by the teacher, which can involve not only sending text messages but any other media at hand on WhatsApp. During this step, the order of the messages sent by the student is not important. Finally, the student can indicate the warm-up is finished with the command “@end", and from that point, the system will ignore messages from the student regarding the warm-up.

From a technical point of view, all media types available in WhatsApp can be used in warm-ups; however, to achieve a simpler interaction and a smaller command set, only one item from each media type are accepted in the activity. For example, if a warm-up involves the students sending a descriptive text, only the last text message sent will be considered for the class results. This way, students can update their submitted responses without any additional command and with the "low cost" of copying and pasting text messages previously sent.

Text messages can also accommodate special markers defined by the teacher, likewise other commands preceded by "@”. Such markers can be used to encourage students to analyze specific points on a warm-up and facilitate the visualization of answers. Spaces and accents are ignored in markers to facilitate their recognition.

For every text or media message sent by students to the mAssingment, from the warm-up selection and student identification until the end of warm-up, the system always returns a feedback message indicating the success or failure of the ongoing action, in addition to instruction for the next steps if necessary.

There are three commands always available for students using the mAssignment. One of them was already mentioned, the finish activity command "@end". The command used to select the warm-up “@<Warm-up_code >”, continues available after the start of the activity, and can be used to resend the warmup instructions. Finally, the status command "@status" can be used anytime to check which items have already being submitted and which items are pending, including markers expected in the text messages.

All the content sent by students to the mAssignment is made available for the teacher and optionally to the students through a Web page. The content is organized as tables, in which the columns differ according to the warm-up settings and each row presents the submission from one student.

\section{Method}

The calls for the next warm-ups were always provided online, at the end of the class, on the Wikispaces platform. Each warm-up call included the estimated effort, the pedagogical goal, a brief contextualization, and instructions. The deadline for submission was between 2 to 7 days, in general, two hours before the next class, allowing the teacher to synthesize the 
results from the exercise and adapt the lesson according to the class's demands.

Throughout the semester, 14 warm-ups were conducted. Two of those were adapted to be carried out using the mAssignment system: the first one adapted was an information collection exercise and the second one was a peer evaluation. The students could choose to do the warm-ups exercises on the Web using the Wikispaces platform and Web forms, or via WhatsApp using mAssignment. It was explained that this choice wouldn't influence their grade. The warm-up's instructions were carefully designed to be as similar as possible in the two modes of interaction, so the content produced by both groups could be comparable. The results from the WhatsApp submissions were published on a Web page jointly with the content from warmups performed using Wikispaces.

After the activities with the proposed system, an informal discussion about the perception of students toward the mAssignment and the warm-up dynamic was conducted, and two researchers took notes of the feedback from the students.

\section{1) Quantitative Data}

All warm-up conducted in the semester had its participation rate recorded; students who did not finish the course were not accounted.

The contributions were measured considering the number of text characters presented in the messages written by students in warm-ups, including markers.

\section{2) Qualitative Data}

The notion of quality of participation in the warm-up's context is somewhat subjective and differs from an evaluation activity because is not directly connected with the correctness of the students' submission, but rather with the effort made by them. We applied clear criteria to assess this attribute for each type of activity; in warm-ups involving an analysis, we checked if all the items requested were fulfilled; in warm-ups with information collection, we observed whether the images submitted were captured or created by the student or only copied from the Internet.

\section{Warm-up Descriptions}

\section{1) AQ12: Simplified Peer Evaluation}

The peer evaluation activity provided in the WhatsApp had as pedagogical goal promoting a first contact with a simplified evaluation of user interface prototypes. The prototypes in this exercise consisted of an image illustrating the main screen of an application for smartphones, as shown in Fig 6, followed by a short description. In the evaluation, students should provide positive points (“@+”), negative points (“@ -”), in addition to questions or suggestions (“@\$”) regarding the other groups" work. The prototypes produced by each group were preregistered in the system and the instructions of the warm-up indicated to students which group should be evaluated.

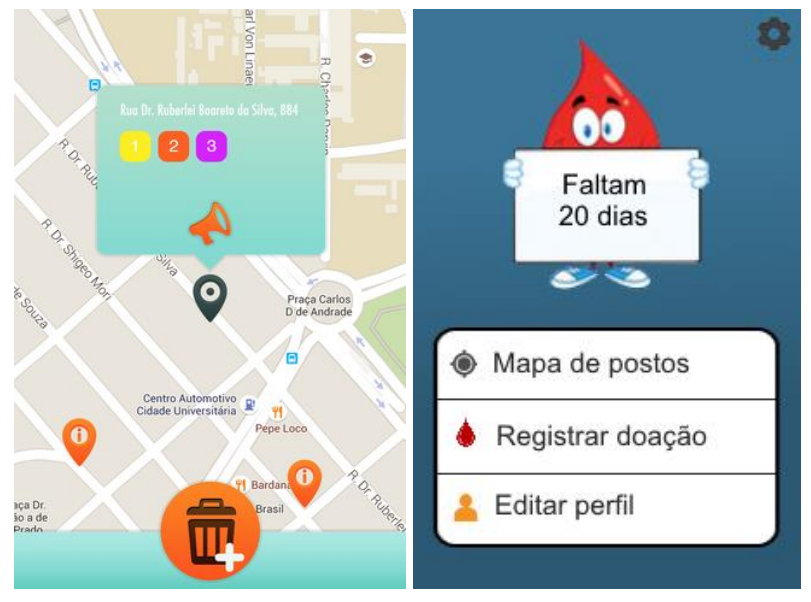

Fig 6. Examples of main screens evaluated in AQ12

\section{2) AQ13: Three levels of good design}

The activity of information collection provided in the WhatsApp had as pedagogical goal expose the students to Norman's Theory of Emotional Design [17]. Students were requested to capture an image from their everyday life using their smartphone or digital camera and analyze how the three levels of design would be applied to the object. The analysis for the visceral level was indicated with the marker "@levell"; the behavioral level was indicated by “@level2”, and the reflexive level by "@level3”.

Fig 7 shows the initial instructions for the AQ12 and AQ13 warm-ups, presented to students right after the login. The interaction with the system always started with a greeting message "Hey <student_login>!" to acknowledge the students that they were correctly identified. The following information varied according to the warm-up and was sent in separate messages to improve the readability and organization of instructions. The second message included the warm-up's name, the link to the full description online and a numbered list of instructions. And the third message listed for the student the global commands available.

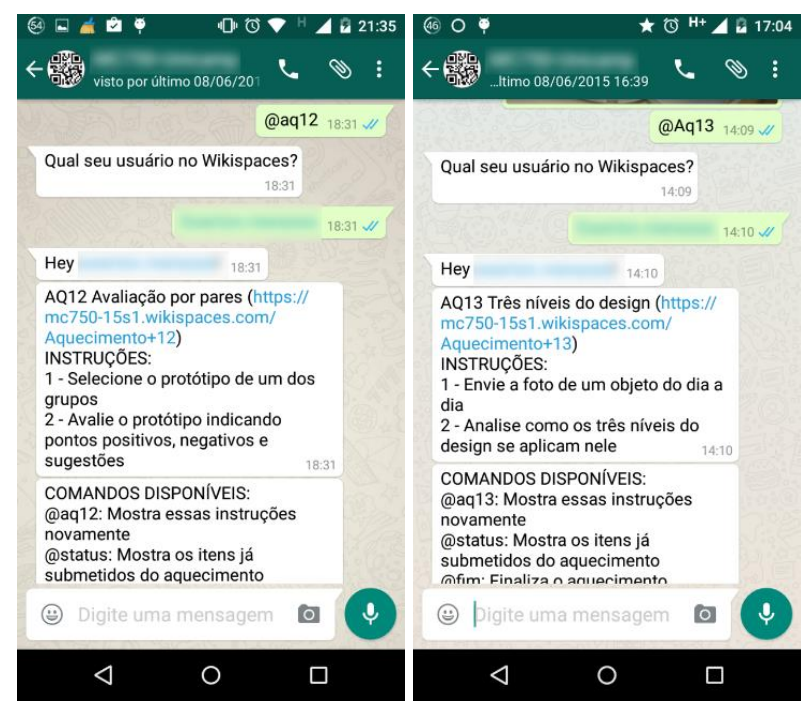

Fig 7. Initial instructions for warm-ups $A Q 12$ and $A Q 13$ 


\section{3) AQ09: Universal Design}

This warm-up was provided only in the Wikispaces and was analyzed in the case study for being an information collection activity very similar to AQ13. The activity's pedagogical goal was to explore the concepts of Accessibility and Universal Design [18] in public spaces. These students were requested to capture an image from the University Campus or other places they go regularly, showing problems or good examples related to Accessibility and Universal Design. Beside the image, it was also required to post the name of the place and a description.

\section{E. Results}

The average participation rate during the semester was $72 \%$ and the graph in Figure 8 shows a significant difference between warm-ups AQ12 and AQ13; in both students had the option to submit their assignment using WhatsApp via the system. While AQ12 had the highest participation rate in the semester, AQ13 was among the activities with lower participation, suggesting that providing the warm-ups also in the WhatsApp did not strongly influence the students' participation. Other factors such as the type of warm-up activity could have played a more important role.

Analyzing the whole semester, it is possible to observe that students' participation had a slight decrease trend $(\rho=-0,5703)$ and, different from expected, the warm-ups' deadline had a low correlation with the participation $(\rho=-0,085)$. Also, the drop in the participation rate in the last two warm-ups can be attributed to the period of exams combined with the fact that after the AQ12 most of the students had already achieved the minimum criteria for approval in the course.

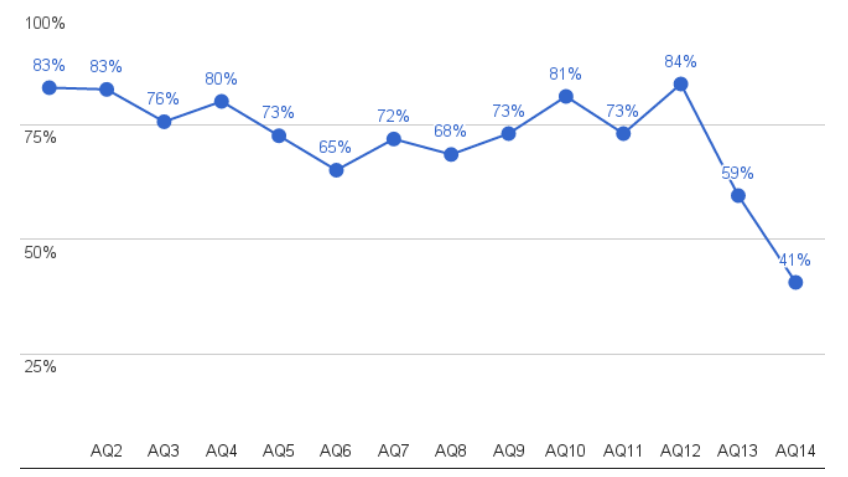

Fig 8. Participation rate during the semester

Despite the decrease in participation rate between AQ12 and AQ13 from $84 \%$ to $59 \%$, the proportion of students that executed the warm-up activity using the WhatsApp didn't change in a significant way (as the graphs in Fig 9 shows), indicating that the type of activity didn't influence the WhatsApp usage. In absolute numbers, six students used WhatsApp in the AQ12 and five in the AQ13. Out of the six students in the AQ12, three kept on using WhatsApp in the AQ13.

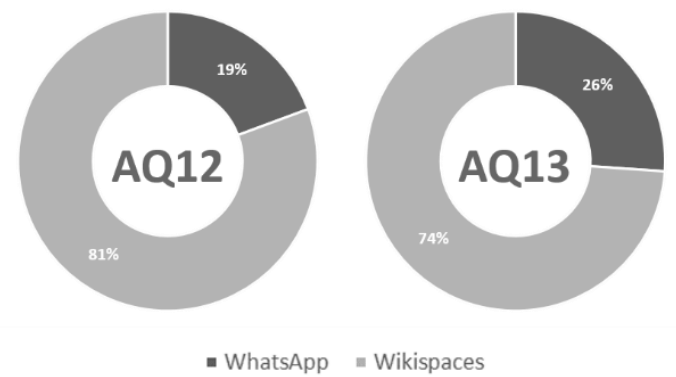

Fig 9. Artifact used by students to perform AQ12 and AQ13

Comparing the content produced by the students in the warm-ups, Table 1 shows the average number of characters in texts receive via WhatsApp was smaller than texts from Wikispace in both warm-ups, although this difference was statistically significant only in the AQ13. (two-sample $\mathrm{t}(18)=$ $2,101, \mathrm{p}=0,006)$. This result suggests that writing on mobile devices influences the size of students' texts, probably due to typing on virtual keyboards of smartphones be slower and more error-prone than on traditional computer keyboards [19]. Besides, students also mentioned the text input in mobile devices as a negative point in the interaction with WhatsApp.

TABLE I. Number of characters in the texts produced in the warm-ups

\begin{tabular}{c|c|c} 
& AQ12 & AQ13 \\
\hline WhatsApp & $589 \pm 257$ characters & $385 \pm 42$ characters \\
Wikispaces & $639 \pm 80$ characters & $654 \pm 75$ characters
\end{tabular}

In AQ12, most students indicate the positive and negative points, and improvement suggestions in the evaluations; only 2 submitted an incomplete warm-up via WhatsApp and 3 via the Wikispaces. An example of a negative point raised from the WhatsApp submissions is "Due to its simplicity, if the description wasn't presented I wouldn't have been able to understand the function of each screen element...". An example of a positive point: "Apparently it is simple to be used. The menu seems to be well explained...". In the AQ13, most students analyzed all three levels of design, with only one student sending an incomplete warm-up via WhatsApp while 8 failed to consider some design level from the selected object via Wikispaces. A good example from a text analyzing the behavioral level of interacting with a computer mouse sent via WhatsApp is "Further to be aligned with the design of a common mouse, this is extremely comfortable and very practical to be transported.". A good example of analysis in the reflexive level: "It transmits a strong message about ourselves to the others. Like the watch shown by Don Norman, it impresses people by distinctive design"

We did not detect relevant grammatical and rhetorical differences, nor any differences related to other linguistic features between messages submitted using WhatsApp and Wikispaces. Particularly in the messages from WhatsApp, we did not find any abbreviation or other Portuguese shortenings often used in this communication app. 
Students finished the warm-ups via WhatsApp exchanging an average of 7 messages with the system (AQ12:SD=1 | $\mathrm{AQ13}: \mathrm{SD}=1,50)$. Considering that to finalize both $\mathrm{AQ} 12$ and AQ13 it was necessary to send at least 4 messages; this number suggests that students had no trouble in understanding the system's instructions and commands to perform the activities via WhatsApp. From all students that tried to perform the warmups, only one left the system without finishing it, the remaining accomplished all steps required to complete the activities; some students used the system via WhatsApp for doing both warmups.

The interactions with a higher number of messages exchanged were from students exploring the system's commands available in the mAssignment, especially the "@status", and from students that needed more than one attempt to enter their login or the warm-up code correctly.

Although the information available in the system log didn't allow to verify the time spent to perform the warm-ups using the system via WhatsApp, the time range between the first and the last message varied from 4 to 38 minutes in AQ12 and from 9 to 31 minutes in AQ13. Considering only students that finished the warm-ups, these time ranges were very close to the estimated time for the activities.

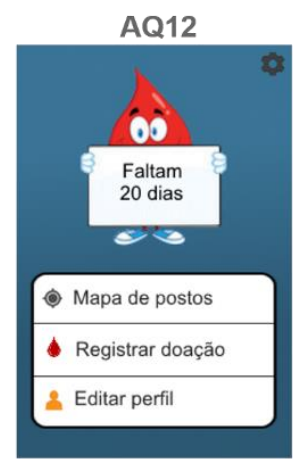

$$
\begin{aligned}
& \text { @+ simple design } \\
& \text { @+ relevant information }
\end{aligned}
$$

@ \$ specify what the message "20 days left" is about, despite being logical what it is about, is better to ensure the user understanding.

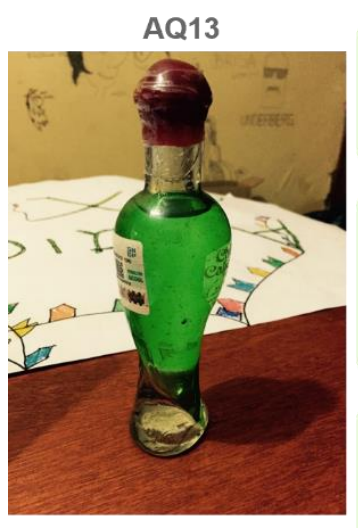

@level1 The image shows a drink bottle that has a simple design, not containing a glow and texture at good levels

@level2 The recipient doesn't stand by its quality since is manufactured with poor quality materials. However, its content stands out by its quality, providing comfort and happiness

@level3 The object usage with its content transmit a sensation of quality and happiness. The object meets the user's expectation.

Fig 10. Examples of warm-up messages received with different usage of markers than expected

Most students sent their analyses in a single message with the three marks as specified in the warm-ups' instructions, they also used line breaks to organize multiple markers. It is worth noting that there were different usages of the markers than the expected as illustrated in Fig 10. In AQ13 one student analyzed the three levels of emotional design using one message for each level, initiating his messages with the corresponding marker; another student used the marker for positive points ("@+") more than once in the same message to distinguish the different positive points that he listed in his peer evaluation.

Analyzing the images submitted in the collecting information activities, while in AQ09, which was not provided by the option to use with mAssignment, (only Wikispaces), 50\% of images sent by students were photos captured by the students themselves. In AQ13 on the other hand, this percentage dropped to $32 \%$ among the students that used Wikispaces and raised to $80 \%$ among students that used WhatsApp, probably by the facility of taking pictures and sending them through their smartphones.

\section{DISCUSSION}

To incorporate mobile communication applications already known in informal contexts into the formal teaching and learning activities, the stakeholders more directly involved (e.g. students, teachers, teaching assistants) need to construct together new meanings for the application. For this process to be effective it is essential that the mobile application usage makes sense not only from a technological point of view but also from a pedagogical point of view.

From the usage viewpoint, it is not enough to conduct a conventional activity in the smartphone. The activity must be designed to leverage the interaction opportunities and unique features of the mobile devices. This fact became clear in our study in the AQ13 warm-up, in which we instructed students to capture a photo from their daily life. This activity 'makes more sense' using a smartphone than using a computer, mainly because the capture and submission of a photo with the proposed system require fewer steps than to capture the image using a digital camera (or the smartphone itself), transfer it to the computer, authenticate with the system, and only then submit the activity.

From the pedagogical perspective, the smartphone is not used only as a "small and ultra-portable computer", but as a device integrated with the course content, and with the diverse and situated contexts of students. Capturing a photo from daily life, as was asked in AQ13, probably stimulates the critical thinking and a more active attitude of students toward the knowledge seen in the class, besides the benefit of learning more than with a common search on the Web for "emotional design examples". Similarly, the evaluation of a mobile application prototype performed in AQ12 on the mobile device itself enables to anticipate design issues that could otherwise go unnoticed.

The educational scenario explored in this study is formal not just because of the activities are mandatory or the students have deadlines to finish it, but also due to the formality introduced in the pedagogical activities. To allow a faster analysis and consequentiality the usage of this data during the class, as defines the pedagogical dynamic of the JiTT, the warm-ups had a well-defined structure, clear grade and acceptance criteria. So instead of asking students only to "comment" or "discuss", the warm-ups requested specific points to be analyzed, e. g. "submit 
at least one positive point, one negative point, and one suggestion" or "analyze the three levels of designs". The system previously described had the challenge to support this formality with its feedback messages, commands, and markers.

The organizational semiotic artifacts (Semiotic Diagram, Evaluation Frame, and Semiotic Ladder) used in the initial phase of this study guided design decisions and helped define design elements to support the shift in meaning of the WhatsApp use. Some design elements of the system enhanced the level of formality explicitly (for example, the use of QR Code as the system profile picture, the course's name in the contact information fields, feedback messages, and messages with instructions and available commands), while others did so implicitly (such as, publication of content posted on the Web to be discussed in the next class). However, other design elements (for example, the message "Hey <student_login>" presented shortly after the user identification) seek to maintain some informal elements of interaction with WhatsApp not to totally strip the its originals characteristics of usage.

The degree of formality promoted by the system made apparent the shift in meaning in relation to WhatsApp, observed in the more formal language without abbreviations and slang used by students in the texts submitted in both warm-ups (even with reports of the difficulties in the typing of long texts with the smartphone virtual keyboard).

The relatively small number of students that performed the warm-ups using WhatsApp and the system is probably related to the period of the semester that the experiment was conducted. Typically, in the end of the semester, students were troubled with a high overload of activities and less inclined to engage in optional exercises. Nonetheless, the results of the interaction with the system (amount of message exchanged, high success rate, positive feedback, and usage of formal written language) suggest the students were successful in meaning construction for the system embedded in WhatsApp.

The mobile learning strategies that encourage students to use their own device raised concerns related to information privacy from students and made mandatory to perform the activities in the mobile device a delicate issue. In this study, the phone number and the profile pictures of students were not published on the Web, only the teacher had access to this information.

Different from other mobile technologies and applications institutionally adopted by universities and schools, which have their usage restricted to formal teaching context, mobile communication applications are already present in informal contexts, from both students and teachers, have better potential to support constructivist models of teaching and learning. However, this appropriation is not trivial, because it involves a shift in meaning and changes in the original purpose of use as shown in the case study. Therefore, we argue that the construction of an effective technical solution needs to reflect both formal aspects of the course dynamics and informal aspects from the mobile application involved.

\section{CONCLUSION}

Mobile devices are increasingly present in the daily life of students and teachers, and the literature indicates countless advantages in their usage in educational contexts. This study explored an approach to include smartphones in pedagogical contexts that instead of introducing new applications, promotes a change in the meaning of applications already used in informal contexts. To investigate how the stakeholders, handle this shift in meaning, we proposed a system that was used by undergraduate students to perform pre-class activities via WhatsApp.

The results of this exploratory study suggest the instructions, rules, and commands defined, in addition to the online publication of the content created with the system could support students in establishing news meanings for WhatsApp as a communication tool used in more formal contexts. Besides, it extended the purpose of usage of the tool, conceived originally as a social, informational and conversational communication tool [20].

In terms of further work, we intend to investigate the shift in meaning of mobile applications conducting other pedagogical activities both outside and inside the classroom, using other multimedia resources of these devices, such as location, audio, video, etc. Furthermore, we also intend to explore a more formal usage of WhatsApp for collaboration itself, including activities involving groups. Finally, we aim to propose mechanisms to facilitate the creation of these pre-class activities and the subsequent integration of the generated content in the class dynamic.

\section{ACKNOWLEDGMENT}

This study was funded by $\mathrm{CNPq}$ funding agency (process \#149997/2014-0 and \#308618/2014-9). We acknowledge also the Institute of Computing and teachers and students involved in the study.

\section{REFERENCES}

[1] M. Sharples, I. Arnedillo-Sánchez, M. Milrad, and G. Vavoula, "Mobile Learning: Small Devices, Big Issues," in Technology-Enhanced Learning, Dordrecht: Springer Netherlands, 2009, pp. 233-249.

[2] C. H. Crouch and E. Mazur, "Peer Instruction: Ten years of experience and results," Am. J. Phys., vol. 69, no. 9, p. 970, 2001.

[3] G. M. Novak, A. Gavrin, and C. Wolfgang, Just-in-time teaching: blending active learning with web technology. Prentice Hall, 1999.

[4] J. Schell, B. Lukoff, and E. Mazur, "Catalyzing Learner Engagement Using Cutting-Edge Classroom Response Systems in Higher Education," in Increasing Student Engagement and Retention Using Classroom Technologies Classroom Response Systems and Mediated Discourse Technologies, C. Wankel, Ed. Emerald, Bingley, 2013, pp. 233-261.

[5] S. Kinash, J. Brand, and T. Mathew, "Challenging mobile learning discourse through research: Student perceptions of Blackboard Mobile Learn and ipads," Australas. J. Educ. Technol., vol. 28, no. 4, pp. 639-655, 2012.

[6] R. Calvo, A. Arbiol, and A. Iglesias, "Are All Chats Suitable for Learning Purposes? A Study of the Required Characteristics," Procedia Comput. Sci., vol. 27, no. Dsai 2013, pp. 251-260, 2014.

[7] J. Gikas and M. M. Grant, "Mobile computing devices in higher education: Student perspectives on learning with cellphones, smartphones \& social media," Internet High. Educ., vol. 19, pp. 18-26, 2013.

[8] E. Kassens-Noor, "Twitter as a teaching practice to enhance active and informal learning in higher education: The case of sustainable tweets," Act. 
Learn. High. Educ., vol. 13, no. 1, pp. 9-21, Mar. 2012.

[9] S. Prestridge, "A focus on students' use of Twitter - their interactions with each other, content and interface," Act. Learn. High. Educ., vol. 15, no. 2, pp. 101-115, Jul. 2014.

[10] D. Bouhnik, M. Deshen, and R. Gan, "WhatsApp Goes to School : Mobile Instant Messaging between Teachers and Students," J. Inf. Technol. Educ. Res., vol. 13, pp. 217-231, 2014.

[11] A. Bere, "A comparative study of student experiences of ubiquitous learning via mobile devices and learner management systems at a South African university," in Proceedings of the 14 th Annual Conference On World Wide Web Applications, 2012, no. November, pp. 4-17.

[12] P. Rambe and A. Bere, "Using mobile instant messaging to leverage learner participation and transform pedagogy at a South African University of Technology," Br. J. Educ. Technol., vol. 44, no. 4, pp. 544-561, Jul. 2013.

[13] A. Fadhil and A. Villafiorita, "An Adaptive Learning with Gamification \& Conversational UIs: The Rise of CiboPoliBot," in Adjunct Publication of the 25th Conference on User Modeling, Adaptation and Personalization, 2017, pp. 408-412.

[14] F. Moreno, E. Manfio, C. R. Barbosa, and J. D. Brancher, "Tical: Chatbot sobre o Atlas Linguístico do Brasil no WhatsApp," in Anais do XXVI Simpósio Brasileiro de Informática na Educação, 2015, no. Sbie, pp. 279288
[15] K. Liu, Semiotics in Information Systems Engineering. New York, NY, USA: Cambridge University Press, 2000.

[16] M. C. C. Baranauskas, "O Modelo Semio-participativo de Design," in Codesign de Redes Digitais: Tecnologia e Educação a Serviço da Inclusão Social, Penso Editora, 2013, pp. 38-66.

[17]D. A. Norman, Emotional Design: Why We Love (or Hate) Everyday Things. Basic Books, 2005.

[18] C. for U. Design, "The Principles of Universal Design," 1997. [Online]. Available: https://www.ncsu.edu/ncsu/design/cud/about_ud/udprinciples.htm. [Accessed: 14-Apr-2016].

[19] E. Hoggan, S. A. Brewster, and J. Johnston, "Investigating the effectiveness of tactile feedback for mobile touchscreens," in Proceeding of the twentysixth annual CHI conference on Human factors in computing systems - CHI '08, 2008, pp. 1573-1582.

[20] K. Church and R. de Oliveira, "What's up with whatsapp? Comparing Mobile Instant Messaging Behaviors with Traditional SMS," in Proceedings of the 15th international conference on Human-computer interaction with mobile devices and services - MobileHCI '13, 2013, pp. $352-361$. 